\title{
Is it Possible to Develop a Language without Exception Handling?
}

\author{
Richa Baijal \\ Student, M.Tech, \\ Computer Science and Engineering \\ Career Point University, \\ Alaniya, Jhalawar Road, \\ Kota-325003 (Rajasthan)
}

\begin{abstract}
In this paper, a knowledge of library files that are used to handle exceptions in different languages is provided. The languages taken $\mathrm{C}, \mathrm{C}++$ and Java are taken as the reference languages. This paper provides the knowledge about library files used to handle exceptions in these languages which are very important from a programmer/researchers'point of view.
\end{abstract}

\section{General Terms}

Library files in $\mathrm{C}, \mathrm{C}++$ and Java ; error handling in $\mathrm{C}$, Exceptions.

\section{Keywords}

Exceptions, Library files in C ,C++,Java; error handling, exception handling.

\section{INTRODUCTION}

Abnormal condition which occurs during runtime during the execution of a program is called an exception/runtime error. Runtime error is in a way different from exception. If there is a runtime error, it is due to some hardware malfunction whereas an exception is caused due to improper logic provided in the program. When such a condition is encountered, it has to be resolved by the language constructs/compilers so that reliability of the language is maintained.

\section{STUDY OF LANGUAGES TO GET AN IDEA ABOUT THE LIBRARY FILES THAT HANDLE EXCEPTIONS/ERRORS}

\subsection{Error Handling In C :}

$\mathrm{C}$ does not provide direct support for error handling . C functions return -1 or NULL when a error occurs in the program. They also set an error code errno which makes a programmer understand that error has occurred in the program . Let us consider an example and then understand the functions and header files associated with it.

Example :

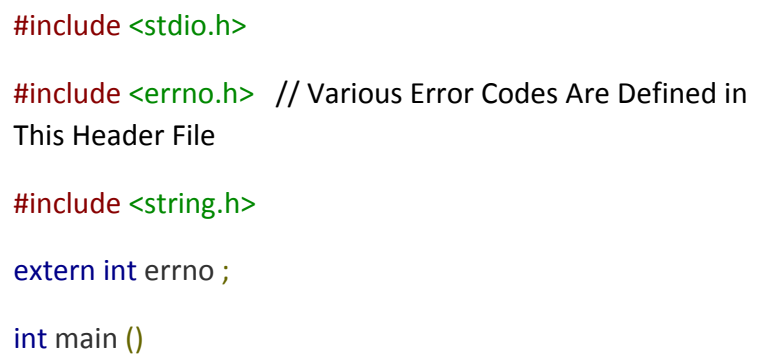

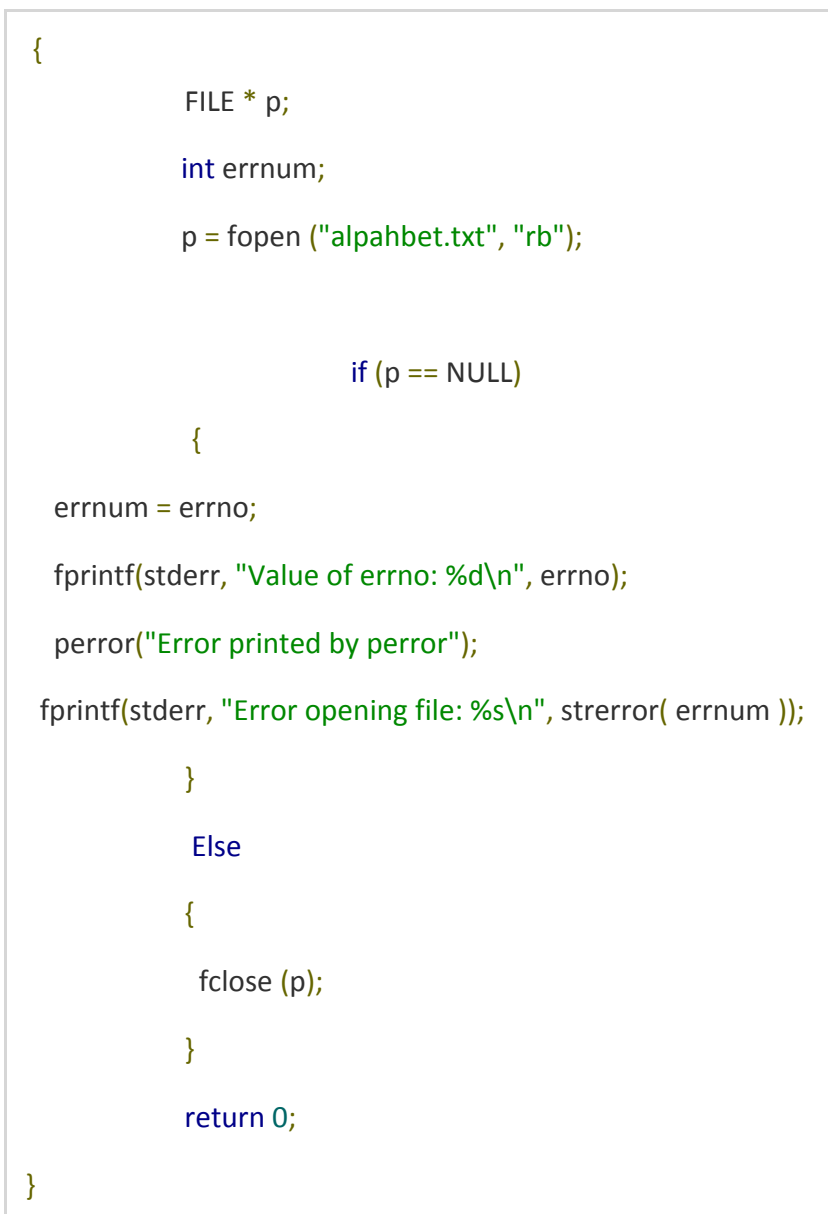

Well, now just have a quick look at the program and let us understand it simply in one line :

This program will return a ' 0 ' if everything goes well i.e the file which $\mathrm{i}$ am looking for is found.

But,unfortunately things don't go well for me. I get the following Output for this program :

Output :

Value of errno : 2

Error printed by perror : No such file or directory

Error opening file: No such file or directory

Pretty Cool ! Now, let us understand how is it achieved in C. 
Well, $\mathrm{C}$ has a header file to handle the errors <errno.h> the same we included while writing our program. This is a library file in $\mathrm{C}$ and contains macros which identify what type of error has occurred by displaying the no. In integer format.Let us have a look at this macros list :

\begin{tabular}{|c|c|c|}
\hline Macros & Error No. & Type of Error \\
\hline \#define EPERM & 1 & $\begin{array}{l}\text { Operation Not } \\
\text { permitted }\end{array}$ \\
\hline \# define ENOENT & 2 & $\begin{array}{l}\text { No such file or } \\
\text { directory }\end{array}$ \\
\hline \# define ESRCH & 3 & No such process \\
\hline \# define EINTR & 4 & $\begin{array}{l}\text { Interrupted system } \\
\text { Call }\end{array}$ \\
\hline \# define EIO & 5 & I/O Error \\
\hline \# define ENXIO & 6 & $\begin{array}{l}\text { No such device or } \\
\text { address }\end{array}$ \\
\hline \# define E2BIG & 7 & $\begin{array}{l}\text { Argument list too } \\
\text { long }\end{array}$ \\
\hline $\begin{array}{ll}\# & \text { define } \\
\text { ENOEXEC } & \end{array}$ & 8 & Exec format error \\
\hline \# define EBADF & 9 & Bad file number \\
\hline \# define ECHILD & 10 & No child processes \\
\hline \# define EAGAIN & 11 & Try again \\
\hline \# define ENOMEM & 12 & Out of memory \\
\hline \# define EACCES & 13 & Permission denied \\
\hline \# define EFAULT & 14 & Bad address \\
\hline $\begin{array}{ll}\# & \text { define } \\
\text { ENOTBLK } & \end{array}$ & 15 & $\begin{array}{ll}\text { Block } & \text { device } \\
\text { required } & \end{array}$ \\
\hline \# define EBUSY & 16 & $\begin{array}{l}\text { Device or resource } \\
\text { busy }\end{array}$ \\
\hline \# define EEXIST & 17 & File exists \\
\hline \# define EXDEV & 18 & Cross device link \\
\hline \# define ENODEV & 19 & No such device \\
\hline \# define ENOTDIR & 20 & Not a directory \\
\hline \# define ISDIR & 21 & Is a directory \\
\hline \# define INVAL & 22 & Invalid argument \\
\hline \# define ENFILE & 23 & File table overflow \\
\hline
\end{tabular}

\begin{tabular}{|l|c|l|}
\hline \# define EMFILE & 24 & $\begin{array}{l}\text { Too many open } \\
\text { files }\end{array}$ \\
\hline \# define ENOTTY & 25 & Not a typewriter \\
\hline \# define ETXTBSY & 26 & Text file busy \\
\hline \# define EFBIG & 27 & File too large \\
\hline \# define ENOSPC & 28 & $\begin{array}{l}\text { No space left on } \\
\text { device }\end{array}$ \\
\hline \# define ESPIPE & 29 & Illegal seek \\
\hline \# define EROFS & 30 & $\begin{array}{l}\text { Read only file } \\
\text { system }\end{array}$ \\
\hline \# define EMLINK & 31 & Too many links \\
\hline \# define EPIPE & 32 & Broken pipe \\
\hline
\end{tabular}

The macro list clearly defines our error code no. 2 i.e. it is unable to find the file we specified.

It says : No such file or directory.

There are 131 such error codes in $\mathrm{C}$ defined under <error.h> library.

Let us quickly examine two more functions and their work in error handling :

1. perror() : It returns the textual value of current errno.

As in our example, error code is 2. Since,we have used the perror() function it is returning the string value for the corresponding error code.

2. Strerror() : It returns the pointer to the textual representation of current errno value.

Stderr is a file stream also called as Standand Error Stream which is used to output all the errors.

\subsection{Exception Handling in $\mathrm{C}++$ :}

Exception is a condition that occurs during runtime. In $\mathrm{C}++$ ,an exception is handled using three keywords or block namely try catch and throw.[2]

1. throw : A program throws an exception when it first encounters it. It shows that the program has encountered a problem.

2. catch : catch represents the catching of an exception. In other words, it specifies the place in the program where you want to handle the problem.

3. try :A try block identifies the block of code for which particular exceptions will be activated.

It is followed by one or more catch blocks.

Let us take a n example to understand Exception Handling in $\mathrm{C}++$ : 


\section{Example:}

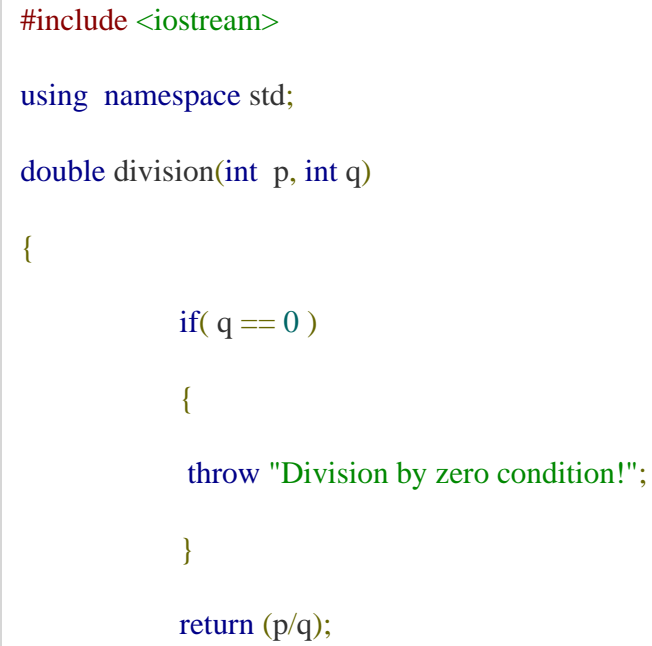

Output : Division by Zero condition !

Since, an exception of type const char* is raised, therefore while catching the exception it is mentioned in catch block .

A short note about using namespace standard routine here :

The std namespace is special; it is short for the word "standard." The built in C++ library routines are kept in the standard namespace. That includes stuff like cout, cin, string, vector, map, etc. Because these tools are used so commonly, it's popular to add "using namespace std" at the top of your source code so that you won't have to type the std:: prefix constantly. And because these functions are kept in a namespace, if you really want to use "vector" as a variable name, you still can. Namespaces give you more freedom to use short, accurate names.

Let us now study what type of exceptions are handled by C++ :[3]

There are standard exceptions which are defined in <exception> header file in $\mathrm{C}++$. They are arranged in parentchild class hierarchy as shown :

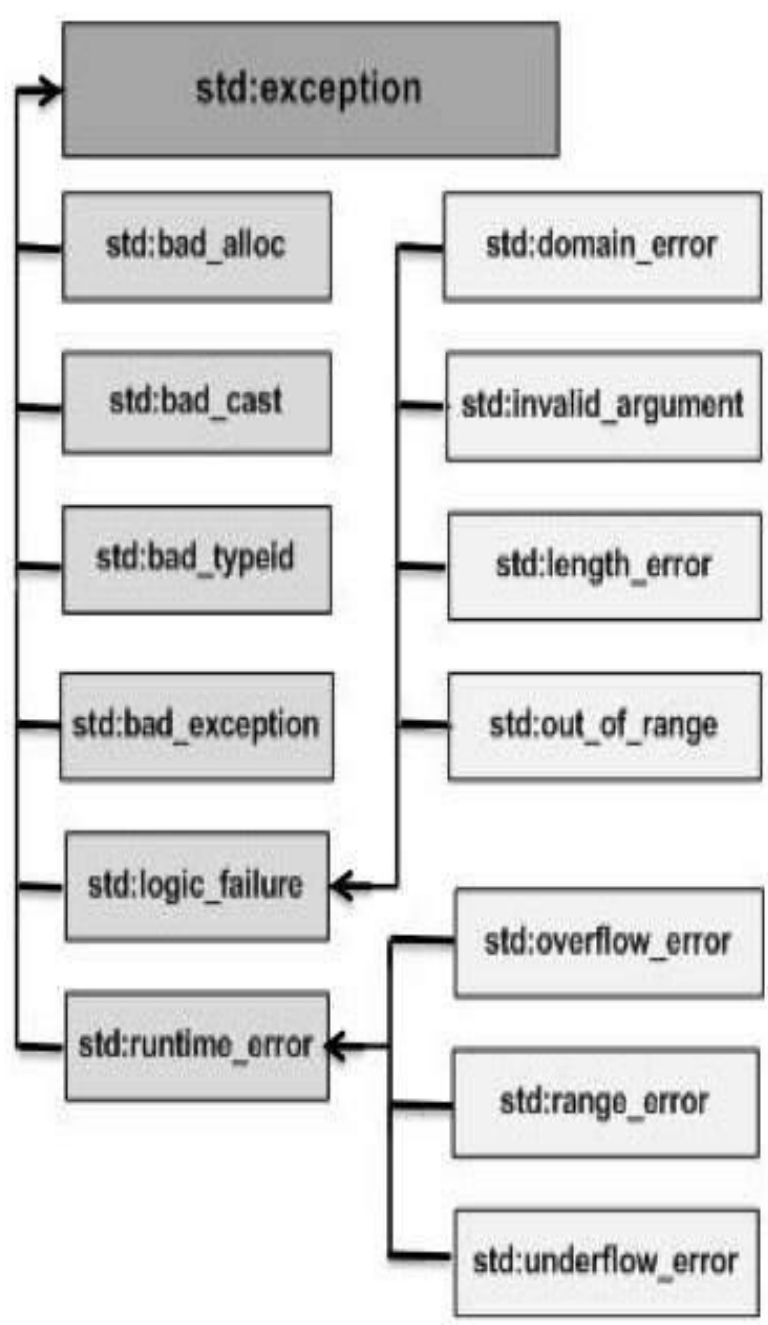

The table provides a definition of these exceptions in brief :

\begin{tabular}{|l|l|}
\hline Exception & Description \\
\hline std::exception & $\begin{array}{l}\text { An exception and parent class of all } \\
\text { the standard C++ exceptions. }\end{array}$ \\
\hline std::bad_alloc & This can be thrown by new. \\
\hline std::bad_cast & $\begin{array}{l}\text { This can be thrown } \\
\text { by dynamic_cast. }\end{array}$ \\
\hline
\end{tabular}




\begin{tabular}{|l|l|}
\hline std::bad_exception & $\begin{array}{l}\text { This is useful device to handle } \\
\text { unexpected exceptions in a C++ } \\
\text { program }\end{array}$ \\
\hline std::bad_typeid & This can be thrown by typeid. \\
\hline std::logic_error & $\begin{array}{l}\text { An exception that theoretically can } \\
\text { be detected by reading the code. }\end{array}$ \\
\hline std::domain_error & $\begin{array}{l}\text { This is an exception thrown when a } \\
\text { mathematically invalid domain is } \\
\text { used }\end{array}$ \\
\hline std::invalid_argument & $\begin{array}{l}\text { This is thrown due to invalid } \\
\text { arguments. }\end{array}$ \\
\hline std::length_error & $\begin{array}{l}\text { This is thrown when a too big } \\
\text { std::string is created }\end{array}$ \\
\hline std::underflow_error & $\begin{array}{l}\text { This is thrown if a mathematical } \\
\text { underflow occurs. }\end{array}$ \\
\hline std::range_error & $\begin{array}{l}\text { This can be thrown by the at } \\
\text { method from for example a } \\
\text { std::vector and } \\
\text { std::bitset<>:::operator[](). }\end{array}$ \\
\hline std::runtime_error & $\begin{array}{l}\text { An exception that theoretically can } \\
\text { store a value detected by reading the code. }\end{array}$ \\
\hline not:overflow_error & $\begin{array}{l}\text { This is thrown if a mathematical } \\
\text { overflow occurs. }\end{array}$ \\
\hline
\end{tabular}

$\mathrm{C}++$ also provides a feature where user can override the exception class and can define a new exception :

Example: Overriding std :: exception class to create your own exception

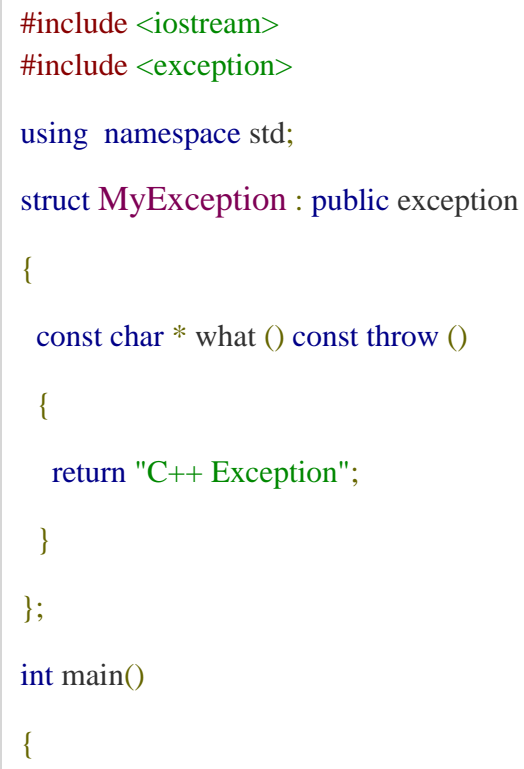

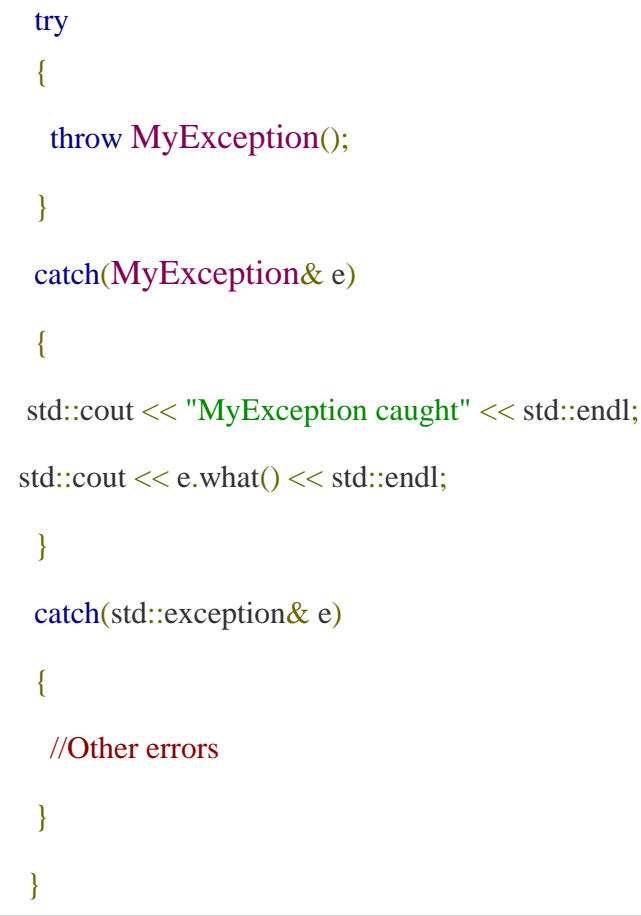

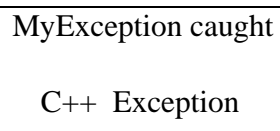

Use of what () : what () is a public method provided by exception class and it has been overridden by all child exception classes. It returns the cause of an exception.

\subsection{Exception Handling in Java} There are two types of exceptions in Java.

1. Checked Exceptions :All exceptions other than runtime exceptions are referred to as Checked Exceptions.

Examples of checked Exceptions :
(i) ClassNotFound Exception
(ii) Illegal AccessException
(iii) NoSuchFieldException
(iv) EOFException, etc.

2. Unchecked Exceptions : All run-time exceptions are called unchecked exceptions.

Examples of unchecked Exception :
(i) Arithmetic Exception
(ii) ArrayIndexOutOfBoundsException
(iii) NullPointer Exception
(iv) NegativeArraySizeException,etc.

Exception Heirarchy in Java :[4,6] 


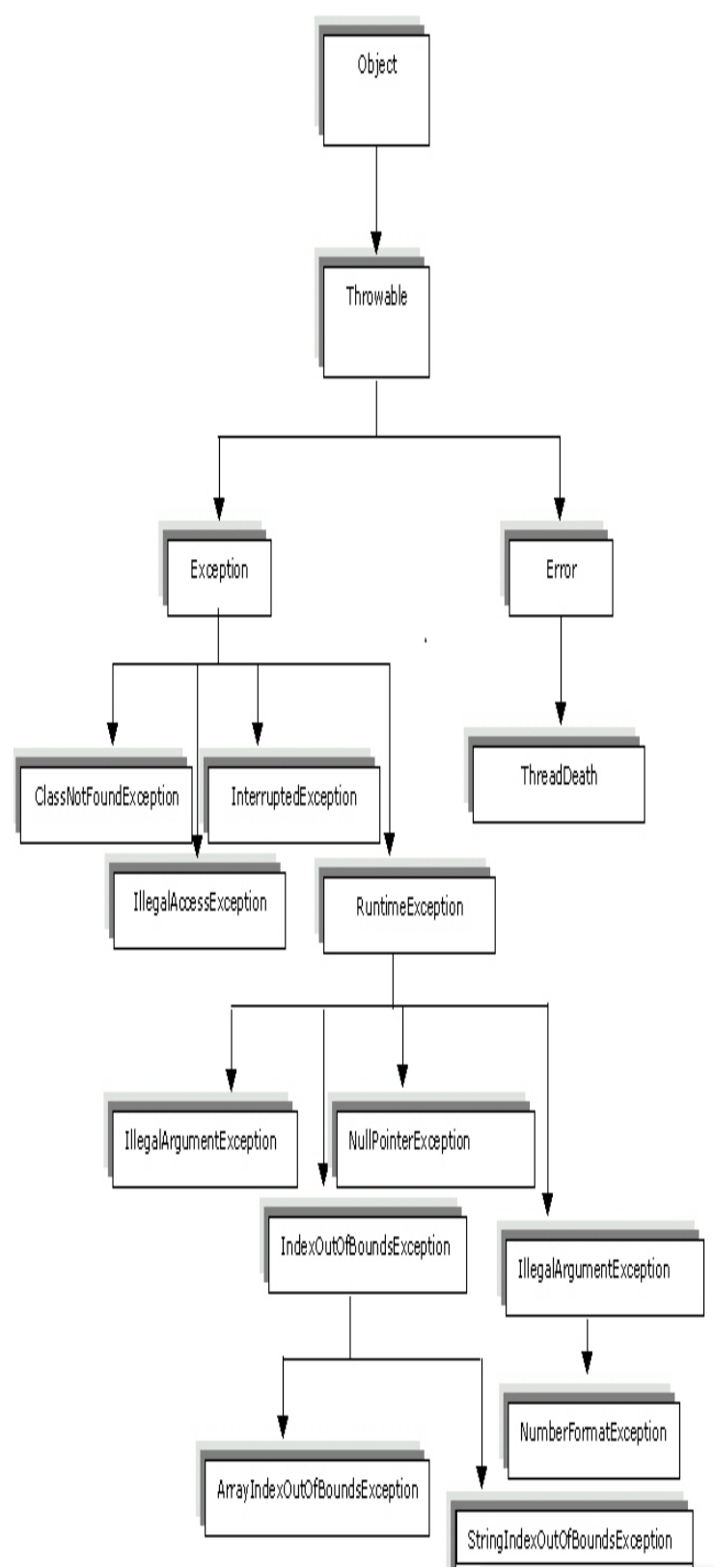

Heirarchy of Java exception Classes :[4,5]

So far,we have seen seen exception classes or macros in $\mathrm{C}++$ and $\mathrm{C}$ respectively. Java is awidely used language with wide no. Of classes defined to handle exceptions. Here is a look:

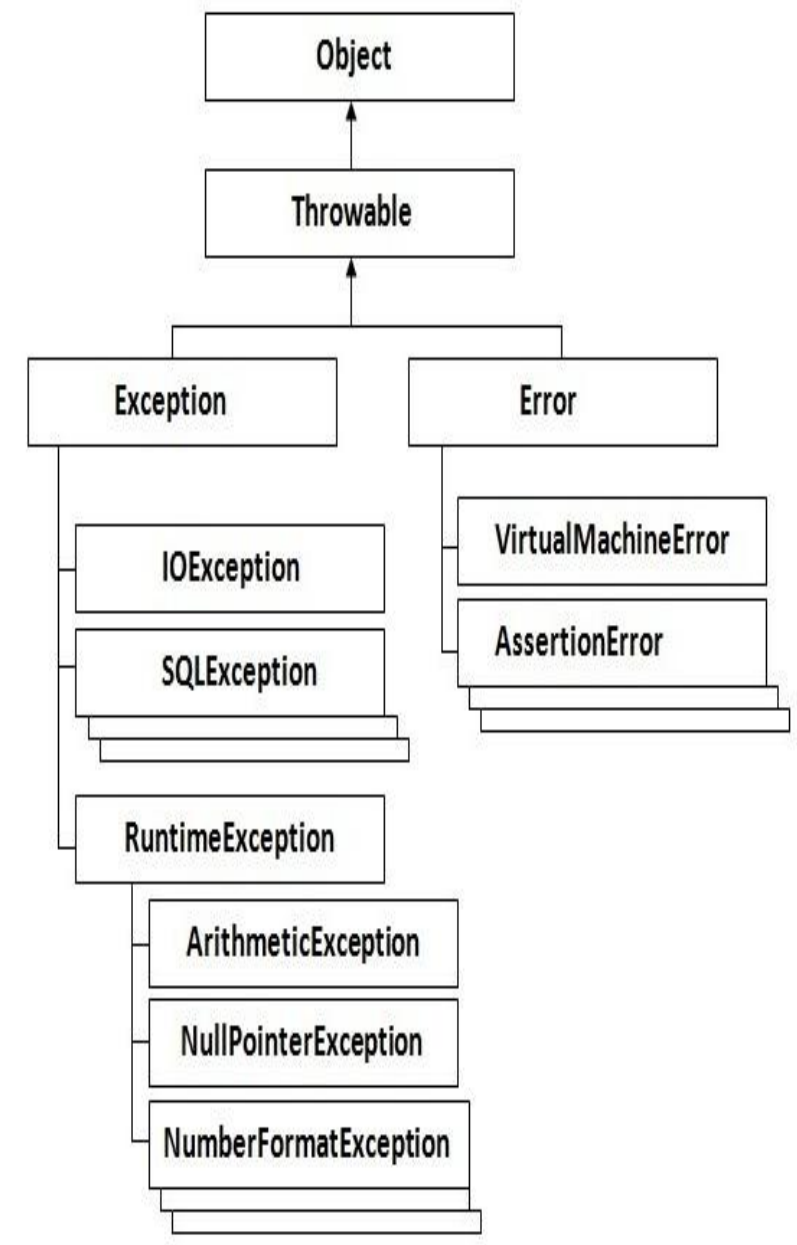

Java uses 5 keywords to handle exceptions.

These are :

1.try:-In try block we enclose the code that might throw an exception.Try block is necessary followed by a catch of finally block in Java.

2.catch:Catch block is used after the try block.It is used to handle the exception.

3.finally : finally block is written after throw/throws block. It is always executed whether an exception occurs or not.

4. throw: It is used to throw an exception that is being caught in the program.

5.throws: If a method cannot handle the checked exception, the method must declare it using throws keyword .

Let us make exception handling in Java more clear by this example:

public class ExcepTest \{

public static void main(String args[] $)\{$

int $\mathrm{a}[]=$ new int[2];

$\operatorname{try}\{$

System.out.println("Access element three :" + a[3]); 


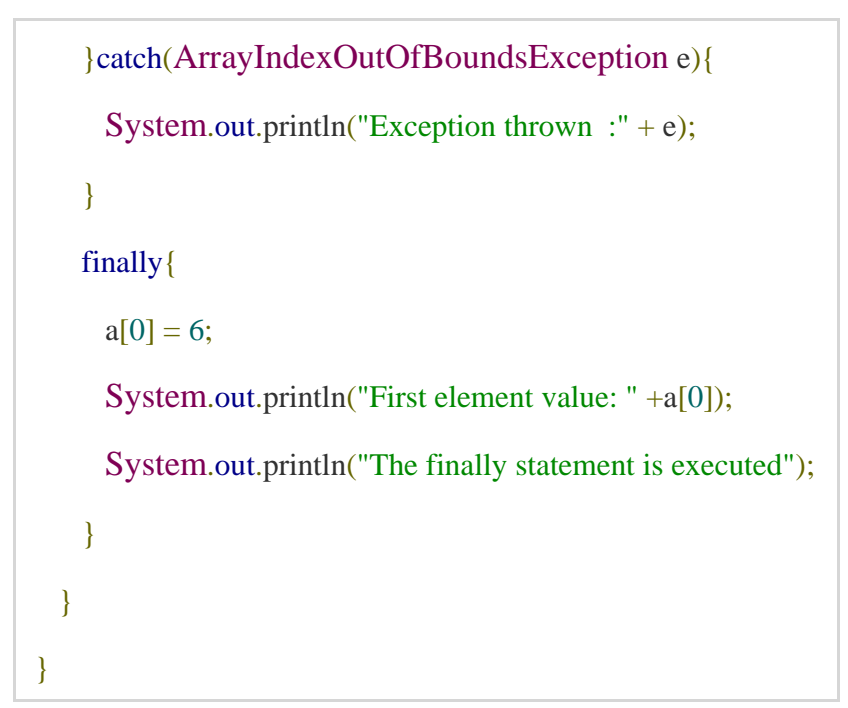

The result of above program will be :

\footnotetext{
Output :

Exception thrown :java.lang.ArrayIndexOutOfBoundsException: 3 First element value: 6

The finally statement is executed
}

\section{User Defined Exceptions :}

Java facilities the programmers to define exception methods themselves. Although the important points to be kept in mind while doing so are :

1. All exceptions must be a child of Throwable class.

2. If you are writing a checked exception,that is automatically enforced by the handle,you should extend the Exception class.

3. If you are writing a runtime Exception, you need to extend the RuntimeException class.

\section{FUTURE AREA OF RESEARCH WORK}

Many modern languages provide rich exception handling features .Three of them,we have gone through. The curiousity revolves around the concept: "Is it possible to design a language without exception handling feature ?".If it is done, what will be gained?

Although, Swift language from Apple does not provides exception handling feature but it does provide assertion and return values which is a kind of error handling mechanism introduced by $\mathrm{C}$.

Why this kind of programming world is desirable ?

Well, a simple answer to this question would be that if processor architecture can be reduced from CISC to RISC ; then why not this feature ? Certain algorithms can definitely enhance this idea of a programming language without exception handling.

\section{CONCLUSION}

Error handling and exception handling library files of different languages have been studied to gain a new lookout of developing a language that will not handle exceptions in future and will seem an equally reliable language then also.

\section{REFERENCES}

[1] Yashavant P. Kanetkar . Let Us C ; Infinity Science Press, 2008

[2] Yashavant P. Kanetkar . Let Us C++ ; Infinity Science Press, 2008

[3] Nicolai M. Josuttis, The C++ Standard Library: A Tutorial And Reference

[4] Stephen Stelting,Robust Java: Exception Handling, Testing, and Debugging; Prentice Hall PTR, 2004.

[5] Herbert Schildt,Java2:The Complete Reference

[6] Bruce Eckel,Thinking In Java;Pearson Education,India. 